\title{
Effect of dietary cholesterol and plant sterol consumption on plasma lipid responsiveness and cholesterol trafficking in healthy individuals
}

\author{
Peter A. S. Alphonse ${ }^{1,2}$, Vanu Ramprasath ${ }^{1,2}$ and Peter J. H. Jones ${ }^{1,2,3 *}$ \\ ${ }^{1}$ Department of Human Nutritional Sciences, University of Manitoba, Winnipeg, MB R3T 2N2, Canada \\ ${ }^{2}$ Richardson Centre for Functional Foods and Nutraceuticals, University of Manitoba, Winnipeg, MB R3T 2N2, Canada \\ ${ }^{3}$ Department of Food Science, University of Manitoba, Winnipeg, MB R3T 2N2, Canada
}

(Submitted 7 April 2016 - Final revision received 28 November 2016 - Accepted 14 December 2016 - First published online 23 January 2017)

\section{Abstract}

Dietary cholesterol and plant sterols differentially modulate cholesterol kinetics and circulating cholesterol. Understanding how healthy individuals with their inherent variabilities in cholesterol trafficking respond to such dietary sterols will aid in improving strategies for effective cholesterol lowering and alleviation of CVD risk. The objectives of this study were to assess plasma lipid responsiveness to dietary cholesterol $v$. plant sterol consumption, and to determine the response in rates of cholesterol absorption and synthesis to each sterol using stable isotope approaches in healthy individuals. A randomised, double-blinded, crossover, placebo-controlled clinical trial ( $n$ 49) with three treatment phases of 4-week duration were conducted in a Manitoba Hutterite population. During each phase, participants consumed one of the three treatments as a milkshake containing $600 \mathrm{mg} / \mathrm{d}$ dietary cholesterol, $2 \mathrm{~g} / \mathrm{d}$ plant sterols or a control after breakfast meal. Plasma lipid profile was determined and cholesterol absorption and synthesis were measured by oral administration of $\left[3,4-{ }^{13} \mathrm{C}\right]$ cholesterol and ${ }^{2} \mathrm{H}-\mathrm{labelled}$ water, respectively. Dietary cholesterol consumption increased total $(0 \cdot 16$ (sem 0.06) mmol/l, $P=0 \cdot 0179$ ) and HDL-cholesterol (0.08 (sEm 0.03 ) $\mathrm{mmol} / \mathrm{l}, P=0.0216$ ) concentrations with no changes in cholesterol absorption or synthesis. Plant sterol consumption failed to reduce LDL-cholesterol concentrations despite showing a reduction $(6 \%, P=0.0004)$ in cholesterol absorption. An over-compensatory reciprocal increase in cholesterol synthesis $(36 \%, P=0.0026)$ corresponding to a small reduction in absorption was observed with plant sterol consumption, possibly resulting in reduced LDL-cholesterol lowering efficacy of plant sterols. These data suggest that inter-individual variability in cholesterol trafficking mechanisms may profoundly impact plasma lipid responses to dietary sterols in healthy individuals.

Key words: Dietary cholesterol: Plant sterols: Plasma lipoproteins: Cholesterol kinetics

Lowering plasma total cholesterol (TC) and LDL-cholesterol levels continue to represent the prime target for CVD risk reduction. Cholesterol absorption and synthesis reciprocally interact with each other to maintain cholesterol homoeostasis in response to dietary perturbations ${ }^{(1-3)}$. However, the magnitude of change in synthesis in response to a change in absorption and vice versa has been shown to be highly variable ${ }^{(4,3)}$. The LDL-cholesterol lowering efficacy of any drug treatment or dietary intervention is affected by this interaction between cholesterol absorption and synthesis ${ }^{(3)}$. Dietary cholesterol is known to raise plasma cholesterol, and variability in such responsiveness exists, where hyper-responders increase plasma TC, being unable to regulate plasma cholesterol homoeostasis either by decreasing intestinal cholesterol absorption or by reducing synthesis ${ }^{(4,5-7)}$. However, hyper-responders represent only about one-fourth of the entire population, whereas the rest have a normal response to cholesterol intake ${ }^{(8,6)}$. In addition, importantly, in these hyper-responders, an increase in both plasma LDL-cholesterol and HDL-cholesterol concentrations is seen ${ }^{(8,9-11)}$. Hence, the current recommendation of $<300 \mathrm{mg} / \mathrm{d}$ of dietary cholesterol for healthy individuals is being questioned $^{(12)}$. Different levels of dietary cholesterol intake have been shown to either modulate ${ }^{(13)}$ or have no effect on cholesterol synthesis ${ }^{(1,14,15)}$. Jones et al. ${ }^{(15)}$ when measured the response of cholesterol biosynthesis in participants to different levels of dietary cholesterol, and modest suppression of cholesterol synthesis by dietary cholesterol was observed independent of plasma cholesterol levels.

In contrast to cholesterol, intake of plant sterols has been repeatedly shown to exert favourable modifications on circulating lipid profile and potential subsequent reduction in risk of CVD ${ }^{(16-19)}$. Typical intakes of plant sterols in the North American diet ranges between 300 and $400 \mathrm{mg} / \mathrm{d}^{(20)}$. Plant sterols are absorbed less $(<2 \%)$ within the intestine compared with cholesterol which is absorbed at approximately $50 \%$ efficiency $^{(21)}$. Plant sterol consumption of about $2 \mathrm{~g} / \mathrm{d}$ has generally been reported to reduce LDL-cholesterol levels by $5-15 \%{ }^{(16,17)}$. Plant sterols are known to lower blood cholesterol

Abbreviations: FSR, fractional synthetic rates; TC, total cholesterol.

* Corresponding author: P. J. H. Jones, email peter_jones@umanitoba.ca 
levels by inhibiting cholesterol absorption, however, the exact mechanism remains to be clarified ${ }^{(16,22)}$. Clinical trials have however shown, similar as seen with cholesterol, large variability among individuals in the degree of LDL-cholesterol lowering response to plant sterol intake ${ }^{(16,19,23-25)}$. LDLcholesterol lowering was found to be greater in individuals with high baseline plasma LDL-cholesterol concentrations compared with those with normal or borderline concentrations ${ }^{(26)}$. Moreover, wide inconsistency in results exists among studies regarding the effect of frequency and time of consumption of plant sterol servings on LDL-cholesterol lowering $^{(23)}$. In addition to lowering cholesterol absorption, plant sterols and stanols are reported to induce a compensatory rise in cholesterol synthesis ${ }^{(17)}$. Inter-individual variations in cholesterol synthesising ability affect the response to LDL-cholesterol lowering efficacy of plant sterols ${ }^{(24)}$. Studies investigating the effects of basal cholesterol synthesis status on LDL-cholesterol lowering efficacy of plant sterols have shown that individuals with high basal cholesterol synthesis exhibit poor LDL-cholesterol lowering response upon plant sterol consumption $^{(24,25)}$. However, a recent study by Ras et al. ${ }^{(27)}$ does not confirm these findings. In addition, genetic variations have been linked to variability in responsiveness to dietary cholesterol and plant sterols ${ }^{(28-30)}$.

The functionality, absorption and metabolism of cholesterol differs from that of plant sterols, despite, only minor structural variances existing between these entities ${ }^{(31)}$. Hence, it is important to understand how individuals respond to dietary cholesterol $v$. plant sterols, given the inherent variabilities in the control and kinetics of cholesterol absorption and synthesis pathways. Such knowledge will aid in developing strategies targeted towards improving the efficacy of cholesterol lowering treatments and thereby mitigating CVD risk. Hence, the present study aims to assess the plasma lipid responsiveness to dietary cholesterol and plant sterols, and to determine the differences in rates of cholesterol absorption and synthesis using stable isotope approaches in healthy individuals.

\section{Methods}

\section{Study participants}

Normocholesterolemic and slightly hypercholesterolemic, but otherwise healthy individuals ( $n$ 55), aged $18-50$ years were recruited from two Hutterite colonies in Manitoba. The two colonies were Barrickman colony located west of Winnipeg city near Cartier, and the Pineland colony located in southeastern Manitoba. Potential confounders such as diet differences, ethnicity and physical activity among the Hutterites are reduced due to the homogenous lifestyle and community living system, providing an opportunity to investigate the study hypotheses in a controlled setting. Otherwise healthy was defined as the absence of a known chronic or infectious disease and supported by the fasting blood sampling. Information sessions were conducted in the colonies and potential study participants were initially screened with a questionnaire regarding personal health information, medical conditions and disease history. Anthropometric parameters such as age, weight, height, blood pressure, hip and waist circumferences were recorded. Individuals determined as potentially eligible underwent a blood screening at the first visit, where a $10 \mathrm{ml}$ fasting blood sample was taken to test for lipid profile measurements such as TC, HDL-cholesterol, LDL-cholesterol and TAG. Inclusion criteria were fasting plasma TAG $<3.0 \mathrm{mmol} / 1$ and a BMI between 20 and $40 \mathrm{~kg} / \mathrm{m}^{2}$. Exclusion criteria included history of recent or chronic use of oral hypolipidemic therapy, probucol, systemic antibodies, corticosteroids, androgens or phenytoin and natural health products including fish oils known to affect lipid metabolism; history of CVD, kidney disease, liver disease, sitosterolemia, recent onset of angina, congestive heart failure, inflammatory bowel disease, pancreatitis, diabetes, or significant gastrointestinal, pulmonary and cancers. Also individuals with a history of chronic use of alcohol ( $>2$ drinks $/ \mathrm{d})$, smoking, exercise with energy expenditure $>16736 \mathrm{~kJ} /$ week ( $>4000 \mathrm{kcal} /$ week) and female individuals who were pregnant or planning for pregnancy during the study period were excluded. A total of fifty-five participants (men=twenty-five; women $=$ thirty) were finally enroled into the study.

\section{Ethical approval and clinical trial registration}

This study was conducted according to the guidelines laid down in the Declaration of Helsinki. The study procedures were evaluated and approved by the Biomedical Research Ethics Board of the University of Manitoba (protocol no. B2013:019). All participants accepted into the study provided written informed consent. The study is registered in the clinicaltrials.gov registry (NCT01825668.).

\section{Study design and protocol}

The study was a free-living, double-blinded, randomised, crossover, placebo-controlled trial conducted at the Nutritional Research Unit of the Richardson Center for Functional Foods and Nutraceuticals (RCFFN), University of Manitoba. An outline of the study design is presented in Fig. 1. The study was comprised of three treatment phases of 4-week duration each, separated by 4 -week-washout intervals, where the test products were not consumed. Participants were randomised to receive three experimental products in the form of milkshakes during the study. All the three experimental milkshake products were isoenergetic (approximately $1292.86 \mathrm{~kJ}$ (309 kcal); total fat: $18.3 \mathrm{~g}$; SFA: $5.85 \mathrm{~g}$; carbohydrates: $22.46 \mathrm{~g}$ and protein: $13.47 \mathrm{~g}$ ) and were prepared in the metabolic kitchen at RCFFN and were then delivered to the two Hutterite colonies twice a week. During the three intervention phases, participants consumed each day a single serving $(240 \mathrm{ml})$ of a milk shake, which contained either $600 \mathrm{mg}$ of cholesterol (egg yolk cholesterol) or a milkshake with $2 \mathrm{~g}$ of plant sterols (from soya containing approximately 55\% $\beta$-sitosterol, 25\% campesterol and $20 \%$ stigmasterol) or a milkshake without plant sterols/cholesterol (control) along with breakfast depending on the phase and assigned treatment. The control and plant sterol incorporated milkshakes contained approximately $30 \mathrm{mg}$ of dietary cholesterol. Supervisors were delegated in each colony to monitor the consumption of milkshakes and compliance to the study 


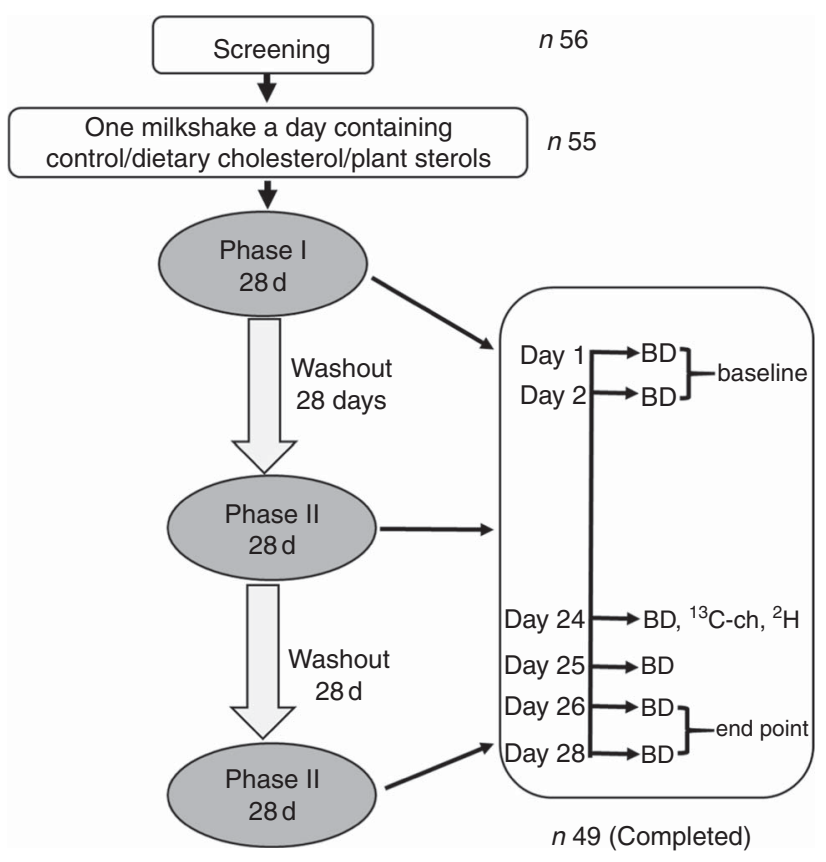

Fig. 1. Study design. BD, blood draws; ${ }^{13} \mathrm{C}$-ch, $\left[3,4-{ }^{13} \mathrm{C}\right]$ cholesterol. ${ }^{2} \mathrm{H},{ }^{2} \mathrm{H}_{2} \mathrm{O}$, ${ }^{2} \mathrm{H}$-labelled water.

protocols. In addition, weekly checklists for milkshake consumption were recorded and collected. Participants were advised to maintain consistency in their typical diet and physical activity levels, and refrain from excessive caffeinated beverages or alcohol during the study phases. Participants completed and returned 3-d food records during the start and end of each phase and were asked to report any major inconsistencies in diet and physical patterns during the intervention period. Moreover, adverse events due to treatments were monitored and participants were advised to communicate to the study personnel regarding any health conditions, symptoms and or change in medications arising during the study period.

\section{Analyses}

Blood collection, sample storage and plasma lipid analyses. Fasting blood samples were collected in heparin containing tubes on days 1, 2, 26 and 28 of each intervention period of the trial. The blood samples after collection were stored at $4{ }^{\circ} \mathrm{C}$ until brought to the laboratory and plasma, buffy coat and erythrocyte fractions were then separated by centrifugation at $3000 \mathrm{rpm}$ for $20 \mathrm{~min}$ at $4^{\circ} \mathrm{C}$. Aliquots of the separated fractions were distributed into different tubes and stored at $-80^{\circ} \mathrm{C}$ until further use. Plasma TC, HDL-cholesterol, TAG and glucose levels were determined by automated enzymatic methods on a Vitros-350 chemistry analyzer (Ortho Clinical Diagnostics). LDL-cholesterol levels were calculated by using the Friedewald equation $^{(32)}$.

Stable isotope tracer intake. On day 24 of each treatment phase, participants consumed $75 \mathrm{mg}$ of the stable carbon isotope $\left[3,4-{ }^{13} \mathrm{C}\right]$ cholesterol dissolved in $5 \mathrm{~g}$ of margarine and spread on half of an English muffin to measure cholesterol absorption. In addition, participants also consumed $0.7 \mathrm{~g} / \mathrm{kg}$ estimated body water (estimated at $60 \%$ of total body weight) of ${ }^{2} \mathrm{H}$-labelled water $\left({ }^{2} \mathrm{H}_{2} \mathrm{O}\right)$ as a tracer to measure cholesterol synthesis. Blood samples were obtained at baseline on day 24 , as well as fasting samples on days 25, 26 and 28 to monitor isotopic enrichment/decay levels.

Determination of cholesterol absorption. Cholesterol absorption was determined using the stable-isotope singletracer method ${ }^{(33)}$. Erythrocyte fraction of blood collected on days 24, 25, 26 and 28 were used for the free cholesterol extraction $^{(34)}$. In brief, about $0.5 \mathrm{~g}$ of erythrocyte fraction was weighed and extracted with $8 \mathrm{ml}$ of methanol at $55^{\circ} \mathrm{C}$ for about $15 \mathrm{~min}$ and to this hexane-chloroform (4:1) mixture was added and vortexed. After vortexing, deionised water was added, vortexed and centrifuged at $1500 \mathrm{rpm}$ for $15 \mathrm{~min}$ at $4^{\circ} \mathrm{C}$. The supernatants were collected and the procedure was repeated with fresh methanol-hexane-chloroform mixture. The pooled supernatants were then dried under $\mathrm{N}_{2}$. The dried supernatants were re-extracted with $0.5 \mathrm{ml}$ of hexane and transferred into GC vials for analysis. These extracts were then used to determine ${ }^{13} \mathrm{C}$-cholesterol enrichment using online GC/combustion/ isotope ratio MS (Agilent 6890N chromatograph interfaced with a Finnigan Delta V Pulse isotope ratio mass spectrometer; IRMS). Isotopic abundances were expressed in $\delta$ per mil (\%o), and were calculated using carbon dioxide as a reference gas and further correction was performed against the Pee Dee Belemnite international standard. From 24 to $96 \mathrm{~h}(72 \mathrm{~h}$ post $-{ }^{13} \mathrm{C}$-cholesterol ingestion), cholesterol absorption is presented as area under the $\left[3,4-{ }^{13} \mathrm{C}\right]$ cholesterol erythrocyte enrichment curve by using the trapezoidal rule and values corrected for baseline values.

Cholesterol synthesis determination. Cholesterol fractional synthetic rates (FSR) $(\% / d)$ were calculated as the rate of incorporation of ${ }^{2} \mathrm{H}$ within the body water pool into erythrocyte cholesterol pools by measuring the ${ }^{2} \mathrm{H}$ erythrocyte enrichment over $24 \mathrm{~h}^{(35)}$. Free cholesterol was extracted as described above for cholesterol absorption ${ }^{(34)}$ and ${ }^{2} \mathrm{H}$ enrichment was measured in both erythrocyte-free cholesterol and plasma water. Erythrocyte-free cholesterol enrichment was measured using online GC-pyrolysis-IRMS (Finnigan Delta V plus Isotope ratio mass spectrometer) using previously established methods. Isotope abundances, expressed in $\delta \%$, were calculated by using $\mathrm{H}_{2}$ as a reference gas. Cholesterol ${ }^{2} \mathrm{H}$ enrichment was expressed relative to standard mean ocean water and a series of standards of known enrichment. Cholesterol FSR represent erythrocyte-free cholesterol ${ }^{2} \mathrm{H}$ enrichment values relative to the corresponding mean plasma water sample enrichment after correcting for the free cholesterol pool. Cholesterol FSR was calculated as shown in the following equation adopted from Jones et $a l .{ }^{(36)}$ and Myrie et al. ${ }^{(37)}$ :

$\operatorname{FSR}(\% / \mathrm{d})=(\delta$ cholesterol $/(\delta$ plasma water $\times 0 \cdot 478)) \times 24 \mathrm{~h} \times 100$.

where $\delta$ is the ${ }^{2} \mathrm{H}$ enrichment of cholesterol or plasma water above baseline in a 24 -h period. The factor 0.478 stands for the 
fraction of $\mathrm{H}_{2}$ atoms per cholesterol molecule that may become enriched by ${ }^{2} \mathrm{H}$ during in vivo cholesterol biosynthesis ${ }^{(36)}$.

The absolute synthesis rate (ASR) of rapidly exchanging free cholesterol was calculated based on previously established models ${ }^{(36-38)}$ :

$$
\operatorname{ASR}(\mathrm{g} / \mathrm{d})=\operatorname{FSR}(\% / \mathrm{d}) / 100 \% \times M 1 \times 0 \cdot 33 .
$$

$M 1$ represents the size of the rapidly exchanging free cholesterol pool and was calculated according to the model of Goodman et $a l^{(38)}$ and the factor 0.33 accounts for the proportion of free cholesterol in the overall plasma TC pool.

Liver function parameters analysis. Analyses of the liver enzymes such alanine transaminase (ALT), aspartate transaminase (AST), alkaline phosphatase (ALKP), $\gamma$-glutamyltransferase (GGT), lactate dehydrogenase (LDH) and total bilirubin (TBIL) as safety biomarkers were performed by autoanalyzer (Vitros 350), with enzymatic reagents.

\section{Statistical analysis}

Sample size was calculated to detect LDL-cholesterol reduction to plant sterol treatment in participants. The sample size was determined to detect an anticipated difference in LDL-cholesterol levels due to plant sterol treatment of $10 \%$ $(0.54 \mathrm{mmol} / \mathrm{l})$ using a SD of $0.732 \mathrm{mmol} / \mathrm{l}^{(39)}$. The $\alpha$ and power were 0.05 and 0.7 , respectively. A sample size of thirty-five participants was calculated with a target of thirty to thirty-one subjects completing the study, taking into account the block size and an estimated $10 \%$ premature participant withdrawal rate. However, to avoid any situation where dropout rates could be higher, and to increase the statistical power of detecting differences in the secondary end points, a sample size of forty-five was calculated. Statistical analysis was performed using the statistical software, SAS version 9.2 (SAS Inc.). Endpoint measurements of treatment and control phases were compared. Values were expressed as least-squares means and standard errors, unless stated. Effects of dietary treatments were examined using a mixed model ANOVA procedure for repeated measures with participant identification code (ID) as a random factor and treatment as an independent factor. The effect of treatment, sequence and sex were included in the model as fixed factors. Statistical significance was set at $P<0.05$ for all analyses. Associations between variables were tested using Pearson's correlation coefficient analyses.

\section{Results}

\section{Baseline characteristics}

Baseline characteristics of the participants who completed the study are presented in Table 1. A total of forty-nine (male = twenty-one; female $=$ twenty-eight $)$ participants completed all the three phases and study requirements. During the first phase, one participant withdrew from the study since they could not adhere to the study's schedule. Another participant withdrew during the second phase of the study because of relocation to another province. In the third phase, two more participants dropped out due to reasons such as conflict with the study and job schedule for one participant and pregnancy planning for the other. We were not able to obtain blood samples from one participant who was absent for the end of the third phase blood sample collection. No change in physical activity was reported by the study participants, and no significant changes in body weight were noticed during the study period. Majority of the participants were normocholesterolemic as indicated by TC levels $(\leq 5.03 \mathrm{mmol} / \mathrm{l})$ and had near optimal LDL-cholesterol levels $(\leq 3.02 \mathrm{mmol} / \mathrm{l})$. A large proportion of the participants were overweight ( $n$ 31; BMI $27-29 \mathrm{~kg} / \mathrm{m}^{2}$ ), and men participants had higher body weights $(P=0 \cdot 0013)$, lower plasma HDL-cholesterol $(P=0.0133)$ and higher TAG $(P=0.0392)$ concentrations compared with women participants.

\section{Plasma lipid responses to dietary cholesterol and plant sterols}

Dietary cholesterol consumption of $600 \mathrm{mg} / \mathrm{d}$ for 4 weeks resulted in an increase in the concentration of plasma TC (0.16 (sEm 0.06) $\mathrm{mmol} / \mathrm{l}, P=0.0179$ ) compared with the control (Table 2). An increase in HDL-cholesterol concentrations (0.08 (sem 0.03) mmol/l, $P=0.0216)$ was found after cholesterol consumption. No changes in TAG $(P=0.9523)$, nonHDL-cholesterol $(P=0.4080)$ concentrations and LDLcholesterol:HDL-cholesterol ratio $(P=0.9993)$ were found following cholesterol consumption. Significant heterogeneity in LDL-cholesterol responsiveness to cholesterol consumption was seen in the study population (Fig. 2(a)). Plant sterol consumption of $2 \mathrm{~g} / \mathrm{d}$ did not show any reductions in plasma TC $(P=0.3327)$, LDL-cholesterol $(P=0.3525)$ and non-HDLcholesterol $(P=0 \cdot 1040)$ concentrations compared with the control. In addition, no changes were seen in plasma TAG $(P=0.6525)$, HDL-cholesterol $(P=0.9801)$ concentrations and LDL-cholesterol:HDL-cholesterol ratio $(P=0.4375)$ following plant sterol consumption when compared with the control. Similar to the effect observed for cholesterol consumption, a large inter-individual variability in LDL-cholesterol responsiveness was found in the study population following plant sterol consumption (Fig. 2(b)). Effects of sex on plasma lipid responses following cholesterol and plant sterol supplementation were analysed (data not shown). Sex significantly influenced the plasma HDL-cholesterol increasing effect of cholesterol consumption in the study population $(P=0.0028)$. Female participants had a greater increase $(0.12$ (SEM 0.04$) \mathrm{mmol} / \mathrm{l}$, $P=0.0424)$ in plasma HDL-cholesterol concentrations in response to cholesterol consumption compared with males (0.05 (sEm 0.05) mmol/l, $P=0.9076)$. No changes in plasma HDL-cholesterol concentrations were seen after plant sterol consumption in either sexes. Moreover, no other significant sex effects were observed with any other plasma lipid variables in response to cholesterol and plant sterol consumption.

\section{Effect of dietary cholesterol and plant sterols on cholesterol absorption and biosynthesis rates}

Consumption of $600 \mathrm{mg} / \mathrm{d}$ of cholesterol for 4 weeks did not result in any changes in cholesterol absorption $(P=0.1192)$ 
Table 1. Baseline characteristics of study participants who completed the study (Least-squares means with their standard errors, $n$ 49)

\begin{tabular}{|c|c|c|c|c|c|c|c|}
\hline \multirow[b]{2}{*}{ Variables } & \multicolumn{2}{|c|}{ Overall ( $n$ 49) } & \multicolumn{2}{|c|}{ Men $(n 21)$} & \multicolumn{2}{|c|}{ Women (n 28) } & \multirow[b]{2}{*}{$P$ values for men $v$. women } \\
\hline & Mean & SEM & Mean & SEM & Mean & SEM & \\
\hline Age (years) & 33.04 & 1.54 & 32.05 & $2 \cdot 51$ & 33.72 & 1.95 & 0.6028 \\
\hline Weight (kg) & $75 \cdot 88$ & 2.33 & $85 \cdot 17$ & 3.82 & 69.48 & 2.32 & 0.0017 \\
\hline $\mathrm{BMI}\left(\mathrm{kg} / \mathrm{m}^{2}\right)$ & 27.81 & 0.75 & $29 \cdot 21$ & 1.29 & 26.83 & 0.87 & 0.1383 \\
\hline Waist circumference $(\mathrm{cm})$ & 86.54 & 1.22 & 94.25 & $2 \cdot 86$ & $80 \cdot 25$ & 2.70 & 0.0042 \\
\hline Hip circumference $(\mathrm{cm})$ & 97.54 & 1.73 & 99.92 & 1.99 & 95.77 & 2.63 & 0.03297 \\
\hline Waist:hip ratio & 0.88 & 0.01 & 0.94 & 0.01 & 0.85 & 0.01 & $<0.0001$ \\
\hline TAG $(\mathrm{mmol} / \mathrm{l})$ & 1.33 & 0.09 & 1.57 & 0.17 & $1 \cdot 18$ & 0.10 & 0.0392 \\
\hline Total cholesterol $(\mathrm{mmol} / \mathrm{l})$ & 5.03 & 0.13 & 5.03 & 0.16 & 5.04 & 0.22 & 0.5208 \\
\hline LDL-cholesterol (mmol/l/) & 3.02 & 0.13 & 3.06 & 0.15 & 2.99 & 0.19 & 0.7596 \\
\hline HDL-cholesterol (mmol/l) & 1.40 & 0.06 & 1.26 & 0.08 & 1.49 & 0.08 & 0.0133 \\
\hline Glucose $(\mathrm{mmol} / \mathrm{l})$ & $5 \cdot 38$ & 0.19 & 5.75 & $0 . .44$ & $5 \cdot 13$ & 0.09 & 0.1822 \\
\hline
\end{tabular}

* $P$ values for men $v$. women by unpaired $t$ test.

Table 2. Plasma lipid responses to dietary cholesterol and plants sterol treatment after 4 weeks ${ }^{\star}$ (Least-squares means with their standard errors, $n$ 49)

\begin{tabular}{|c|c|c|c|c|c|c|}
\hline \multirow[b]{2}{*}{ Lipid variables } & \multicolumn{2}{|c|}{ Control } & \multicolumn{2}{|c|}{ Cholesterol } & \multicolumn{2}{|c|}{ Plant sterols } \\
\hline & Mean & SEM & Mean & SEM & Mean & SEM \\
\hline Total cholesterol (mmol/l) & $4 \cdot 87^{\mathrm{a}}$ & 0.14 & $5.03^{b}$ & 0.13 & $4.78^{\mathrm{a}}$ & 0.14 \\
\hline Absolute change from control & & & 0.16 & 0.06 & -0.09 & 0.07 \\
\hline Change from control (\%) & & & 4.76 & 1.42 & -1.58 & 1.43 \\
\hline LDL-cholesterol (mmol/l) & $2 \cdot 85^{a, b}$ & 0.13 & $2 \cdot 96^{\mathrm{a}}$ & 0.13 & $2 \cdot 73^{\mathrm{b}}$ & 0.13 \\
\hline Absolute change from control & & & 0.11 & 0.06 & -0.12 & 0.06 \\
\hline Change from control (\%) & & & 5.40 & $2 \cdot 61$ & $-2 \cdot 86$ & 2.39 \\
\hline HDL-cholesterol (mmol/l) & $1.44^{\mathrm{a}}$ & 0.06 & $1.53^{\mathrm{b}}$ & 0.06 & $1.45^{\mathrm{a}}$ & 0.06 \\
\hline Absolute change from control & & & 0.09 & 0.03 & 0.01 & 0.03 \\
\hline Change from control (\%) & & & $6 \cdot 64$ & $2 \cdot 06$ & 1.28 & 1.85 \\
\hline Non-HDL-cholesterol (mmol/l) & $3 \cdot 43^{a, b}$ & 0.14 & $3.51^{a}$ & 0.14 & $3.32^{\mathrm{b}}$ & 0.14 \\
\hline Absolute change from control & & & 0.08 & 0.06 & -0.10 & 0.06 \\
\hline Change from control (\%) & & & $4 \cdot 17$ & 1.99 & -2.54 & 1.89 \\
\hline TAG $(\mathrm{mmol} / \mathrm{l})$ & 1.25 & 0.11 & 1.27 & 0.11 & 1.29 & 0.11 \\
\hline Absolute change from control & & & 0.01 & 0.06 & 0.04 & 0.06 \\
\hline Change from control (\%) & & & 1.55 & $3 \cdot 12$ & 7.95 & 4.97 \\
\hline LDL-cholesterol:HDL-cholesterol & $2 \cdot 13$ & 0.13 & $2 \cdot 13$ & 0.13 & 2.05 & 0.13 \\
\hline Absolute change from control & & & -0.002 & 0.06 & -0.08 & 0.06 \\
\hline Change from control (\%) & & & 0.90 & 3.37 & -8.63 & 4.95 \\
\hline
\end{tabular}

${ }^{a, b}$ Mean values with unlike superscript letters are significantly different from each other; $P<0.05$ is considered significant.

* Differences between treatments were assessed by SAS mixed-procedure ANOVA with Tukey-Kramer adjustments.

as measured by the area under the $\left[3,4{ }^{13} \mathrm{C}\right]$ cholesterol erythrocyte enrichment curve compared with the control (Fig. 3 (A)). However, plant sterol consumption reduced cholesterol absorption $(P=0.0004)$ compared with the control (Fig. 3(A)). Compared with control, dietary cholesterol feeding did not affect cholesterol FSR $(P=0.8438)$, which is representative of the proportion of the rapidly turning over cholesterol pool synthesised per day, measured by estimating ${ }^{2} \mathrm{H}$ incorporation after ${ }^{2} \mathrm{H}_{2} \mathrm{O}$ consumption (Fig. 3(B)). On the contrary, plant sterol consumption markedly increased cholesterol FSR $(P=0 \cdot 0026)$ compared with the control (Fig. 3(B)). A similar trend was found where plant sterol consumption increased cholesterol ASR $(P=0.0010)$ compared with the control, however no change was seen after cholesterol consumption $(P=0 \cdot 9131)$. Cholesterol absorption and FSR in response to plant sterols were negatively correlated $(r-0.36, P=0.0166)$ as shown in Fig. 4. When the participants were categorised into groups based on their plasma
LDL-cholesterol responses (hyper- or hypo-responders) to dietary cholesterol and plant sterol supplementation, no changes in cholesterol absorption or cholesterol FSR were observed between the different groups were observed.

\section{Liver function test parameters in response to dietary cholesterol and plant sterols}

No changes in any of the plasma liver enzymes (ALT, AST, ALKP, GGT, LDH and TBIL) were found because of dietary cholesterol or plant sterol consumption compared with the control.

\section{Discussion}

Increased dietary cholesterol absorption usually suppresses endogenous cholesterol synthesis as opposed to the reduction 
(a)

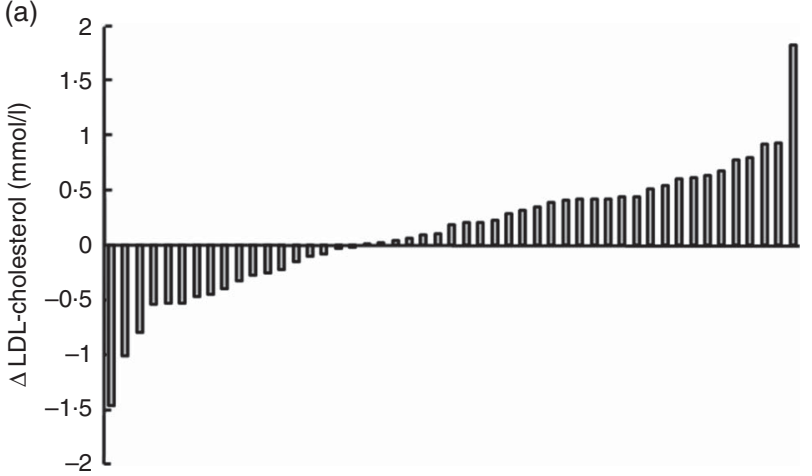

(b)

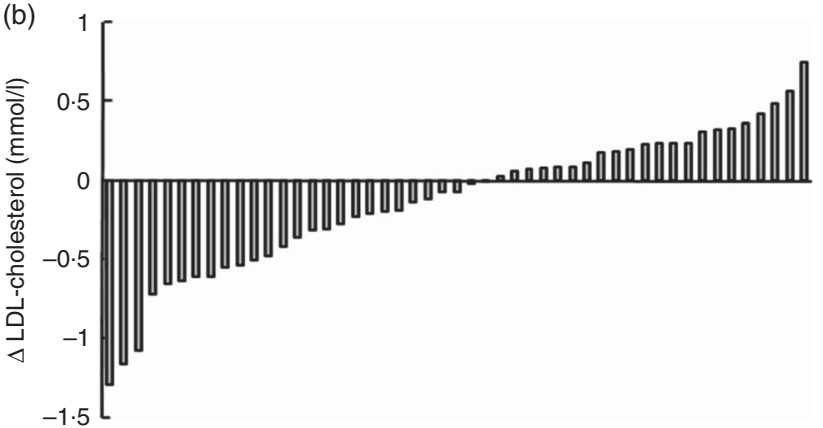

Fig. 2. (a) Individual changes in LDL-cholesterol in response to dietary cholesterol consumption. (b) Individual changes in LDL-cholesterol in response to plant sterol consumption. $\Delta$, Absolute change in LDL-cholesterol $(\mathrm{mmol} / \mathrm{l})$ of end points of dietary cholesterol and plant sterol phases from end point of control phase. End points were mean plasma LDL-cholesterol concentrations of blood draws on days 26 and 28.

of cholesterol absorption and reciprocal elevation of endogenous synthesis caused by plant sterol consumption ${ }^{(2,3,15,17)}$. The present study, using stable isotopes techniques, has shown that a cholesterol challenge of $600 \mathrm{mg} / \mathrm{d}$ did not affect cholesterol absorption or synthesis, but resulted in increased plasma TC and HDL-cholesterol concentrations, whereas, plant sterol consumption of $2 \mathrm{~g} / \mathrm{d}$ induced an over-compensatory increase in cholesterol synthesis corresponding to a small decrease in cholesterol absorption, and possibly subdued the plasma LDL-cholesterol lowering efficacy of plant sterols in healthy Hutterite individuals.

Dietary supplementation of $600 \mathrm{mg} / \mathrm{d}$ of cholesterol for 4 weeks failed to produce any change in plasma LDL-cholesterol concentrations in the study population, however did increase TC concentrations, similar to the observations of various other studies ${ }^{(6,9-11,40,41)}$. These studies support the existence of inter-individual differences in LDL-cholesterol concentration changes leading to hypo- and hyperresponsiveness to dietary cholesterol as seen in our study population. On the other hand, cholesterol supplementation resulted in a significant elevation in plasma HDL-cholesterol concentrations in study participants. A similar effect has been demonstrated by a number of other studies as well ${ }^{(41-44)}$. One interesting finding was that women alone experienced an increase in plasma HDL-cholesterol concentrations to cholesterol consumption regardless of baseline differences in HDL-cholesterol concentrations between men and women. Clifton \& Nestel $^{(45)}$ have reported a similar finding where a cholesterol challenge of $650 \mathrm{mg} / \mathrm{d}$ for 3 weeks together with a low-fat diet resulted in substantial elevation in plasma HDL-cholesterol and large HDL-subclass concentrations in women compared with men ${ }^{(45,46)}$. Such differences have been ascribed to the differential fat distribution between men and women ${ }^{(46)}$. The exact mechanism causing such increase in HDL-cholesterol concentrations remains unknown. Notwithstanding this, it can be inferred that dietary cholesterol consumption does not unfavourably modify plasma lipid profiles in healthy individuals and may endow women with anti-atherogenic benefits by increasing plasma HDL-cholesterol concentrations in comparison with men. Hence, development of sex-specific dietary cholesterol recommendations may prove to be useful.

Surprisingly, plant sterol consumption of $2 \mathrm{~g} / \mathrm{d}$ did not induce a significant reduction in plasma LDL-cholesterol concentrations and TC concentrations in this study population. Contrary to the well-established plasma LDL-cholesterol lowering effect induced by plant sterol intake in numerous studies ${ }^{(18,21,37,47,48)}$, our study did not detect a prominent reduction. Some studies have previously reported the lack of efficacy of plant sterol treatment in lowering cholesterol ${ }^{(49-51)}$. The lack of effects in some of these studies appears to be because of poor solubility of the plant sterol formulations tested ${ }^{(49,50)}$. However, we believe that the plant sterol formulation or food matrix used in our study could have not contributed to the lack of effects, as other studies using similar plants sterol enriched study products have demonstrated substantial LDL-cholesterol lowering efficacy $^{(52-54)}$. Frequency and time of administration have also been suggested as possibly affecting the efficacy of plant sterol formulations $^{(23,51,55)}$. Our study used a single-dose administration of plant sterol treatment accompanied with breakfast, which led us to speculate whether the lack of efficacy stems from dosage and time of administration. But, single dosage studies of plant sterols have shown equivalent efficacies in plasma LDL-cholesterol reduction ${ }^{(37,56,57)}$ comparable with studies distributed with dosage regimens ${ }^{(47,48,58-60)}$. Hence, it does not appear that the lack of efficacy could be because of the single dosage regimen of plant sterols in our study. Cholesterol biosynthesis is known to be affected by circadian rhythm as shown by the modulatory effects of meal time lags on cholesterol synthesis ${ }^{(3,51,61-63)}$. Lower cholesterol absorption during the morning due to the reciprocal relation between absorption and synthesis could potentially render plant sterols ineffective in furthering cholesterol inhibition leading to sub-optimal plasma LDL-cholesterol reduction ${ }^{(51)}$. Doornbos et $a{ }^{(55)}$ showed a single dose consumption of plant sterol formulation with lunch to be more effective in lowering plasma LDL-cholesterol concentrations than when taken before breakfast ${ }^{(55)}$. Moreover, AbuMweis et al. ${ }^{(64)}$ demonstrated that a three-time daily dose of plant sterols were more efficacious in lowering LDL-cholesterol than a single breakfast dose. In contrast, some studies demonstrated optimal plasma LDL-cholesterol lowering with single-dose regimen of plant sterols accompanied with breakfast ${ }^{(25,65,66)}$. Also, unavailability of data on the circadian rhythm of cholesterol absorption makes it difficult to ascribe lack of efficacy to plant sterol products based on the time of ingestion. 
(A)

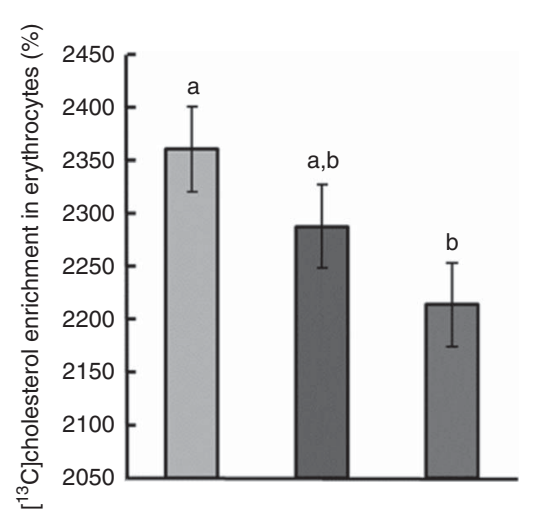

(B)

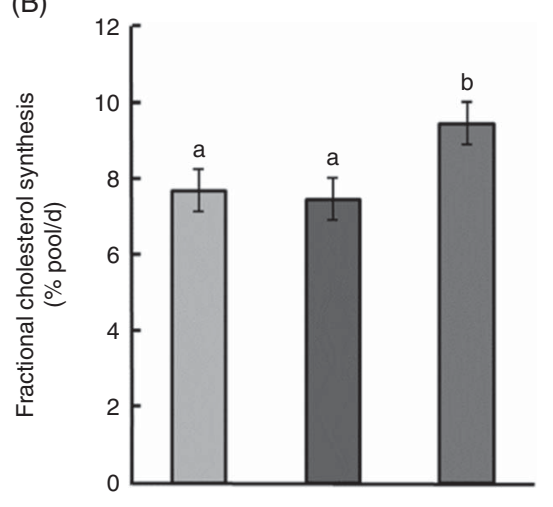

(C)

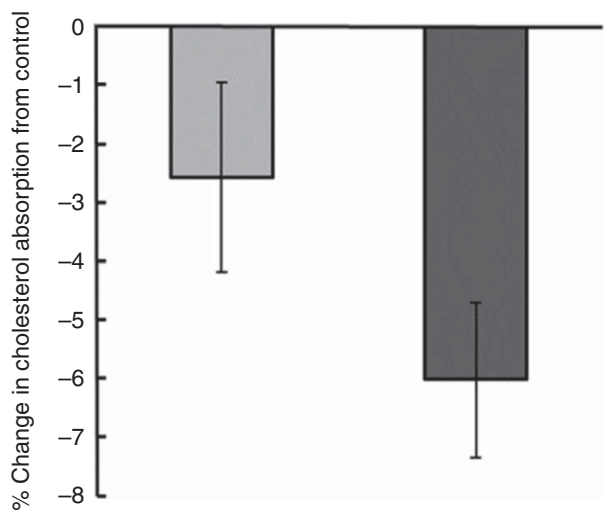

(D)

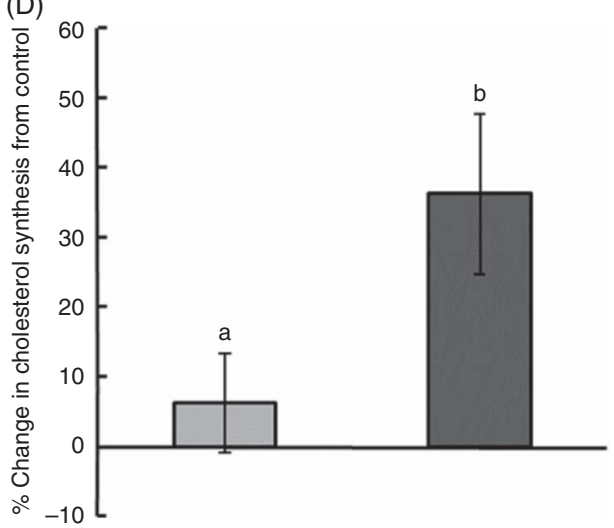

Fig. 3. (A) Cholesterol absorption measured by area under the $\left[{ }^{13} \mathrm{C}\right]$ cholesterol enrichment of erythrocytes over at 24,48 and $96 \mathrm{~h}$ post administration of $75 \mathrm{mg}$ of the stable carbon isotope $\left[3,4-{ }^{13} \mathrm{C}\right]$ cholesterol curve in response to control, dietary cholesterol and plant sterol supplementation phases. (B) The cholesterol fractional synthesis rate measured as the rate of ${ }^{2} \mathrm{H}$ incorporation within the body water pool into erythrocyte cholesterol pools over $24 \mathrm{~h}$ post ${ }^{2} \mathrm{H}_{2} \mathrm{O}$ administration. (C) Percentage change in cholesterol synthesis from control in response to dietary cholesterol and plant sterol consumption. (D) Percentage change in cholesterol absorption from control in response to dietary cholesterol and plant sterol consumption. Values are least-squares means, with their standard errors. Differences between treatments assessed by SAS mixed procedure ANOVA with Tukey-Kramer adjustments. A,B: $\square$, Control; $\square$, dietary cholesterol; $\square$, plant sterol; C,D: $\square$, dietary cholesterol; $\square$, plant sterol. ${ }^{\mathrm{a}, \mathrm{b}}$ Labelled means with unlike letters are significantly different $(P<0.05)$.

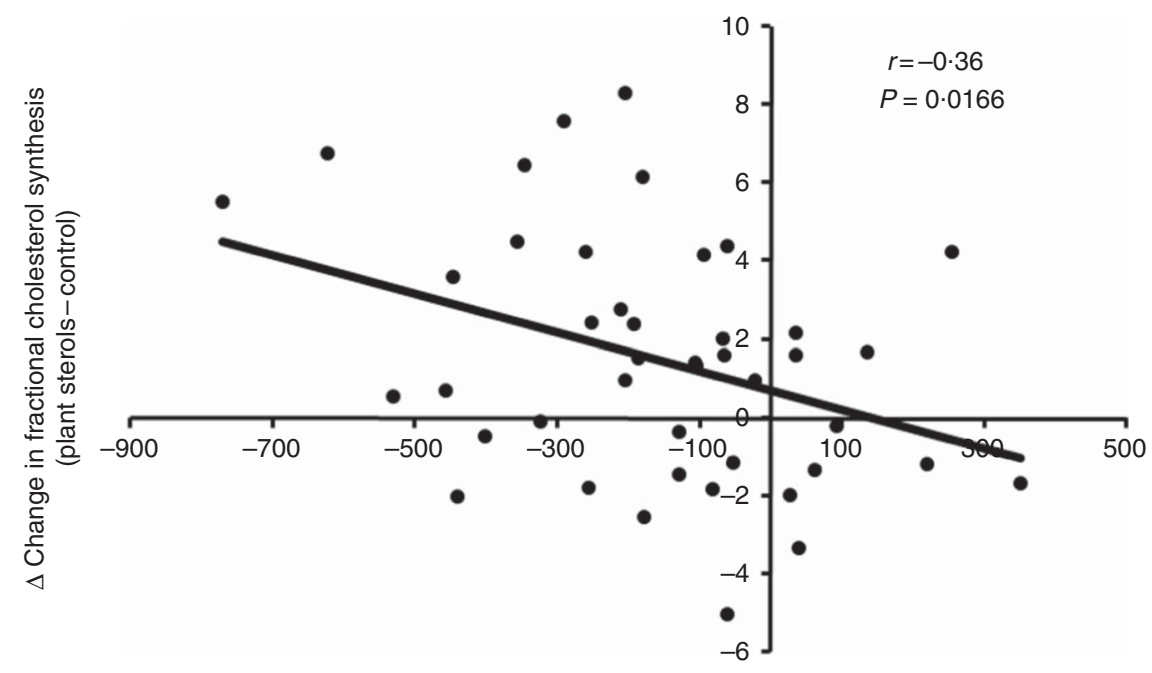

$\Delta$ Change in cholesterol absorption (plant sterols-control)

Fig. 4. Pearson's product-moment correlation $(r)$ between change in cholesterol absorption measured by area under the $\left[3,4-{ }^{13} \mathrm{C}\right]$ cholesterol erythrocyte enrichment curve in erythrocytes $(\%)$ from control and change in fractional cholesterol synthesis (\% pool/d) from control in response to plant sterol consumption. Values are least-squares means, with their standard errors. $P<0.05$ considered significant. $\Delta$, Absolute change. 
Various other factors could also have contributed to the low efficacy of plant sterols in plasma LDL-cholesterol reduction in our study. Large inter-individual variability in plasma LDL-cholesterol responsiveness to plant sterols was present in the population akin to hypo- and hyper-responsiveness to dietary cholesterol. Noteworthy is that our study population was predominantly normocholesterolemic with most individuals exhibiting near-optimal baseline plasma LDL-cholesterol concentrations. It has been shown that individuals with high baseline plasma LDL-cholesterol concentrations experience greater reductions in LDL-cholesterol due to plant sterol or stanol consumption compared with individuals with optimal or borderline LDL-cholesterol concentrations ${ }^{(16,67-69)}$. Another factor to be considered is the cholesterol synthesising ability of the study population. Rideout et al. ${ }^{(24)}$ and Mackay et al. ${ }^{(25)}$ have demonstrated the influence of basal cholesterol synthesis status on the plasma LDL-cholesterol lowering efficacy of plant sterols. These authors have postulated that high basal cholesterol synthesising individuals exhibit poor LDL-cholesterol lowering response upon plant sterol consumption and have assigned a genetic basis for the non-responsiveness ${ }^{(24,25)}$. Mackay et $a l^{(30)}$ proposed that individuals having a combination of $T T$ allele for cholesterol 7-alpha hydroxylase (CYP7A1) rs3808607 SNP and apoE (APOE) $\varepsilon 3$-isoform tend to be non-responders to plant sterol consumption. The present study was conducted in a genetically isolated Hutterite population. It is possible that genetic makeup of the Hutterite participants could have resulted in the non-responsiveness phenotype, imparting low efficacy to plant sterol treatment. The genotype frequency and distribution of various gene polymorphisms affecting cholesterol metabolism is different in the Hutterites compared with the general population ${ }^{(70,71)}$. Therefore, genotyping the study population and testing for associations of candidate SNP affecting lipid and cholesterol metabolism are both crucial in unravelling the observed huge variability in responsiveness to dietary sterols.

Dietary cholesterol consumption did not affect cholesterol absorption as measured by the area under the $\left[3,4-{ }^{13} \mathrm{C}\right]$ cholesterol erythrocyte enrichment curve using the stable-isotope single-tracer method. It is important to consider that the singletracer method does not measure the absolute amount of cholesterol absorbed, but rather represents only the efficiency of cholesterol absorption ${ }^{(21,64)}$. Also, the stable-isotope tracer administered could have been diluted by the dietary cholesterol influx of $600 \mathrm{mg} / \mathrm{d}$, and as only fractional cholesterol absorption happens in the intestine (approximately 50\%), the fraction of the tracer absorbed might have been less than that usually absorbed during the control phase. An increase in plasma TC concentrations was seen following cholesterol challenge, which indicates that the cholesterol absorption was indeed more compared with the control. Using a dual-isotope method specifically for measuring the effect of dietary cholesterol on cholesterol absorption would likely have been more precise in estimating absolute cholesterol absorption. On the other hand, plant sterol administration for 4 weeks reduced cholesterol absorption compared with the control, however, did not reduce plasma LDL-cholesterol concentrations as expected. Similar observation has been previously reported by AbumWeis et al. ${ }^{(64)}$, where the authors found a significant reduction in cholesterol absorption due to plant sterol consumption, but did not see any plasma LDLcholesterol reduction. The authors indicated that a reduction in cholesterol absorption efficiency may not always be associated with a reduction in the magnitude of plasma cholesterol concentrations $^{(64)}$.

In our study, the dietary cholesterol challenge failed to affect cholesterol FSR, measured by estimating ${ }^{2} \mathrm{H}$ incorporation after ${ }^{2} \mathrm{H}_{2} \mathrm{O}$ consumption. Jones et al. ${ }^{(15)}$ did find a modest decrease in synthesis in response to different levels of dietary cholesterol feeding. However, unlike their findings, we did not find any changes in cholesterol synthesis. Concomitantly, we found a marked rise in plasma cholesterol. Nestel \& Poyser ${ }^{(13)}$ observed that moderate intakes of dietary cholesterol induced the suppression of synthesis and prevented the rise of plasma cholesterol. This finding possibly explains the rise in plasma cholesterol concentrations following the cholesterol challenge in our study population and is also indicative of the presence of a large proportion of hyper-responding individuals to dietary cholesterol, as discussed previously. Plant sterol consumption dramatically increased cholesterol FSR and ASR compared with the control presently. A reciprocal up-regulation of cholesterol synthesis in response to a decrease in cholesterol absorption is usually seen ${ }^{(17,37,64)}$. In our study, the increase in synthesis was $36 \%$ (FSR; Fig. 3(D)) and 39\% (ASR) higher compared with the control corresponding to only a $6 \%$ reduction in cholesterol absorption (Fig. 3(C)). Previous studies have reported a larger reduction (approximately 25\%) in cholesterol absorption with a compensatory increase in synthesis ${ }^{(17,37,64)}$. A hyper-reactive increase in cholesterol synthesis in tandem with a small reduction in absorption in our study could have most likely contributed to the reduced effect of plant sterol-induced LDLcholesterol lowering. As previously discussed, the works of Rideout et al. ${ }^{(2)}$ and Mackay et al. ${ }^{(25)}$ on basal cholesterol synthesis status and LDL-cholesterol responsiveness to plant sterols support this observation. Hence, the role of gene polymorphisms affecting cholesterol kinetics and gene-diet interactions determining responsiveness to dietary sterols needs to be better understood ${ }^{(30)}$.

Some of the major strengths of the study include the following. This is the first undertaking to our knowledge where the first direct comparison between the effects of cholesterol $v$. plant sterol intake for 4 weeks on plasma lipids and cholesterol kinetics using stable isotope approaches were examined in a single study. The study was a double-blinded, randomised, crossover, placebo-controlled clinical trial. Another unique aspect was the study population, which was a genetically isolated founder population of Hutterites with minimal environmental and lifestyle-related confounders. However, our study did suffer from some limitations. The study was free-living with less control over dietary intake. However, to our advantage the Hutterites have a community dining system with all individuals in the population consuming typically the same diet, except for individual variations in energy intake. Another limitation of the study is that challenges exist in extrapolating the findings to the general population.

In summary, using stable isotopic assessment in healthy Hutterite individuals, we found that dietary cholesterol 
supplementation did not affect cholesterol absorption or synthesis, but increased plasma TC and HDL-cholesterol concentrations, whereas, plant sterol consumption resulted in an over-compensatory elevation in cholesterol synthesis pertaining to a small reduction in cholesterol absorption. Such hyperreactive increase in cholesterol synthesis corresponding to a miniscule reduction could have possibly hampered the plasma LDL-cholesterol lowering efficiency of plant sterol administration. Our results thereby affirm the existence of inter-individual variability in endogenous cholesterol synthesis, potentially inflecting the reciprocal relationship between cholesterol absorption and synthesis, and the extent to which they are correspondingly offset due to dietary perturbations. Our findings also show that dietary cholesterol consumption did not unfavourably alter the plasma lipid profile of our study participants. Notably, large inter-individual variability in plasma lipid responsiveness to the consumption of dietary sterols was found in the study population. Hence, scrutinising genetic heterogeneity and phenotype associations will facilitate understanding gene-diet interactions that modulate the reciprocal relationship between cholesterol absorption and synthesis, and thereby determine an individual's responsiveness to cholesterol $v$. plant sterol consumption. Development of personalised dietary intervention strategies will therefore become possible which can be directed towards regulating plasma cholesterol levels and prevention of CVD risk.

\section{Acknowledgements}

The authors sincerely thank Sam Guo (Nutragenius Inc.) for donating the plant sterols. The authors also thank the study participants for their engagement. The authors are grateful to Stephanie Haidi, Shuo Yang, Julia Rempel for their assistance with conducting the clinical trial and Haifeng Yang, Dylan MacKay, Elliot Desilets, Yanan Wang and Mohammed Abdullah for their technical assistance.

This study was supported by the Dairy Farmers of Canada and the Agri-Food Research and Development Initiative (grant no. ARDI GRWFWD ARDI 12-1191). The funding agencies had no role in the design, analysis or writing of this article. P. J. H. J. has received funding from the Dairy Farmers of Canada, Danone Inc. and Agriculture and Agri-Food Canada. P. A. S. A. was a recipient of the University of Manitoba Graduate Fellowship and the Manitoba Graduate Scholarship.

The authors' responsibilities were as follows: P. J. H. J., P. A. S. A. and V. R. P.: designed the research; P. A. S. A.: conducted the research, analysed the data and wrote the manuscript; and P. A. S. A., V. R. P. and P. J. H. J.: had primary responsibility for the final content. All authors participated in editing and approving the final manuscript.

The authors declare that there are no conflicts of interest.

\section{References}

1. Grundy SM, Ahrens EH Jr \& Davignon J (1969) The interaction of cholesterol absorption and cholesterol synthesis in man. J Lipid Res 10, 304-315.

2. Santosa S, Varady KA, AbuMweis S, et al. (2007) Physiological and therapeutic factors affecting cholesterol metabolism: does a reciprocal relationship between cholesterol absorption and synthesis really exist? Life Sci $\mathbf{8 0}, 505-514$.

3. Alphonse PA \& Jones PJ (2016) Revisiting human cholesterol synthesis and absorption: the reciprocity paradigm and its key regulators. Lipids 51, 519-536.

4. Quintao E, Grundy SM \& Ahrens EH Jr (1971) Effects of dietary cholesterol on the regulation of total body cholesterol in man. J Lipid Res 12, 233-247.

5. McNamara DJ, Kolb R, Parker TS, et al. (1987) Heterogeneity of cholesterol homeostasis in man. Response to changes in dietary fat quality and cholesterol quantity. J Clin Invest 79, 1729-1739.

6. Barona J \& Fernandez ML (2012) Dietary cholesterol affects plasma lipid levels, the intravascular processing of lipoproteins and reverse cholesterol transport without increasing the risk for heart disease. Nutrients $\mathbf{4}, 1015$.

7. Weggemans RM, Zock PL \& Katan MB (2001) Dietary cholesterol from eggs increases the ratio of total cholesterol to high-density lipoprotein cholesterol in humans: a metaanalysis. Am J Clin Nutr 73, 885-891.

8. Fernandez ML (2010) Effects of eggs on plasma lipoproteins in healthy populations. Food Funct 1, 156-160.

9. Herron KL, Vega-Lopez S, Conde K, et al. (2002) Premenopausal women, classified as hypo- or hyperresponders, do not alter their LDL/HDL ratio following a high dietary cholesterol challenge. J Am Coll Nutr 21, 250-258.

10. Herron KL, Vega-Lopez S, Conde K, et al. (2003) Men classified as hypo- or hyperresponders to dietary cholesterol feeding exhibit differences in lipoprotein metabolism. J Nutr 133, 1036-1042.

11. Greene CM, Zern TL, Wood RJ, et al. (2005) Maintenance of the LDL cholesterol:HDL cholesterol ratio in an elderly population given a dietary cholesterol challenge. J Nutr $\mathbf{1 3 5}$, 2793-2798.

12. Fernandez ML \& Calle M (2010) Revisiting dietary cholesterol recommendations: does the evidence support a limit of $300 \mathrm{mg} / \mathrm{d}$ ? Curr Atheroscler Rep 12, 377-383.

13. Nestel PJ \& Poyser A (1976) Changes in cholesterol synthesis and excretion when cholesterol intake is increased. Metabolism 25, 1591-1599.

14. Wilson JD \& Lindsey CA Jr (1965) Studies on the influence of dietary cholesterol on cholesterol metabolism in the isotopic steady state in man. J Clin Invest 44, 1805-1814.

15. Jones PJ, Pappu AS, Hatcher L, et al. (1996) Dietary cholesterol feeding suppresses human cholesterol synthesis measured by deuterium incorporation and urinary mevalonic acid levels. Arterioscler Thromb Vasc Biol 16, 1222-1228.

16. Demonty I, Ras RT, van der Knaap HC, et al. (2009) Continuous dose-response relationship of the LDL-cholesterollowering effect of phytosterol intake. J Nutr 139, 271-284.

17. Jones PJ, Raeini-Sarjaz M, Ntanios FY, et al. (2000) Modulation of plasma lipid levels and cholesterol kinetics by phytosterol versus phytostanol esters. J Lipid Res 41, 697-705.

18. Lichtenstein AH \& Deckelbaum RJ (2001) AHA Science Advisory. Stanol/sterol ester-containing foods and blood cholesterol levels. A statement for healthcare professionals from the Nutrition Committee of the Council on Nutrition, Physical Activity, and Metabolism of the American Heart Association. Circulation 103, 1177-1179.

19. Ras RT, van der Schouw YT, Trautwein EA, et al. (2015) Intake of phytosterols from natural sources and risk of cardiovascular disease in the European Prospective Investigation into Cancer and Nutrition-the Netherlands (EPIC-NL) population. Eur J Prev Cardiol 22, 1067-1075.

20. Moreau RA, Whitaker BD \& Hicks KB (2002) Phytosterols, phytosterols, and their conjugates in foods: structural diversity, 
quantitative analysis, and health-promoting uses. Prog Lipid Res 41, 457-500.

21. Ostlund RE Jr, Racette SB, Okeke A, et al. (2002) Phytosterols that are naturally present in commercial corn oil significantly reduce cholesterol absorption in humans. Am J Clin Nutr $\mathbf{7 5}$, 1000-1004.

22. De Smet E, Mensink RP \& Plat J (2012) Effects of plant sterols and stanols on intestinal cholesterol metabolism: suggested mechanisms from past to present. Mol Nutr Food Res $\mathbf{5 6}$ 1058-1072.

23. Abumweis SS, Barake R \& Jones PJ (2008) Plant sterols/stanols as cholesterol lowering agents: a meta-analysis of randomized controlled trials. Food Nutr Res 52, 10.3402/fnr.v52i0.1811.

24. Rideout TC, Harding SV, Mackay D, et al. (2010) High basal fractional cholesterol synthesis is associated with nonresponse of plasma LDL cholesterol to plant sterol therapy. Am J Clin Nutr 92, 41-46.

25. Mackay DS, Gebauer SK, Eck PK, et al. (2015) Lathosterol-tocholesterol ratio in serum predicts cholesterol-lowering response to plant sterol consumption in a dual-center, randomized, single-blind placebo-controlled trial. Am J Clin Nutr 101, 432-439.

26. Naumann E, Plat J, Kester AD, et al. (2008) The baseline serum lipoprotein profile is related to plant stanol induced changes in serum lipoprotein cholesterol and triacylglycerol concentrations. J Am Coll Nutr 27, 117-126.

27. Ras RT, Koppenol WP, Garczarek U, et al. (2016) Increases in plasma plant sterols stabilize within four weeks of plant sterol intake and are independent of cholesterol metabolism. Nutr Metab Cardiovasc Dis 26, 302-309.

28. Herron KL, McGrane MM, Waters D, et al. (2006) The ABCG5 polymorphism contributes to individual responses to dietary cholesterol and carotenoids in eggs. I Nutr 136, $1161-1165$.

29. Hofman MK, Weggemans RM, Zock PL, et al. (2004) CYP7A1 A-278C polymorphism affects the response of plasma lipids after dietary cholesterol or cafestol interventions in humans. J Nutr 134, 2200-2204.

30. MacKay DS, Eck PK, Gebauer SK, et al. (2015) CYP7A1rs3808607 and APOE isoform associate with LDL cholesterol lowering after plant sterol consumption in a randomized clinical trial. Am J Clin Nutr 102, 951-957.

31. Gylling H \& Simonen P (2015) Phytosterols, phytostanols, and lipoprotein metabolism. Nutrients 7, 7965-7977.

32. Friedewald WT, Levy RI \& Fredrickson DS (1972) Estimation of the concentration of low-density lipoprotein cholesterol in plasma, without use of the preparative ultracentrifuge. Clin Chem 18, 499-502.

33. Wang Y, Vanstone CA, Parsons WD, et al. (2004) Validation of a single-isotope-labeled cholesterol tracer approach for measuring human cholesterol absorption. Lipids 39, 87-91.

34. Folch J, Lees M \& Sloane Stanley GH (1957) A simple method for the isolation and purification of total lipides from animal tissues. J Biol Chem 226, 497-509.

35. Jones PJ (1990) Use of deuterated water for measurement of short-term cholesterol synthesis in humans. Can I Physiol Pharmacol 68, 955-959.

36. Jones PJ, Ausman LM, Croll DH, et al. (1998) Validation of deuterium incorporation against sterol balance for measurement of human cholesterol biosynthesis. J Lipid Res 39, $1111-1117$.

37. Myrie SB, Mymin D, Triggs-Raine B, et al. (2012) Serum lipids, plant sterols, and cholesterol kinetic responses to plant sterol supplementation in phytosterolemia heterozygotes and control individuals. Am J Clin Nutr 95, 837-844.
38. Goodman DS, Smith FR, Seplowitz AH, et al. (1980) Prediction of the parameters of whole body cholesterol metabolism in humans. J Lipid Res 21, 699-713.

39. Nigon F, Serfaty-Lacrosniere C, Beucler I, et al. (2001) Plant sterol-enriched margarine lowers plasma LDL in hyperlipidemic subjects with low cholesterol intake: effect of fibrate treatment. Clin Chem Lab Med 39, 634-640.

40. Beynen AC, Katan MB \& Van Zutphen LF (1987) Hypo- and hyperresponders: individual differences in the response of serum cholesterol concentration to changes in diet. Adv Lipid Res 22, 115-171.

41. Harman NL, Leeds AR \& Griffin BA (2008) Increased dietary cholesterol does not increase plasma low density lipoprotein when accompanied by an energy-restricted diet and weight loss. Eur J Nutr 47, 287-293.

42. Mutungi G, Ratliff J, Puglisi M, et al. (2008) Dietary cholesterol from eggs increases plasma HDL cholesterol in overweight men consuming a carbohydrate-restricted diet. J Nutr 138, 272-276.

43. Mayurasakorn K, Srisura W, Sitphahul P, et al. (2008) Highdensity lipoprotein cholesterol changes after continuous egg consumption in healthy adults. J Med Assoc Thai 91, 400-407.

44. Blesso CN, Andersen CJ, Barona J, et al. (2013) Whole egg consumption improves lipoprotein profiles and insulin sensitivity to a greater extent than yolk-free egg substitute in individuals with metabolic syndrome. Metabolism 62, 400-410.

45. Clifton PM \& Nestel PJ (1992) Influence of gender, body mass index, and age on response of plasma lipids to dietary fat plus cholesterol. Arterioscler Thromb 12, 955-962.

46. Clifton PM, Abbey M, Noakes M, et al. (1995) Body fat distribution is a determinant of the high-density lipoprotein response to dietary fat and cholesterol in women. Arterioscler Thromb Vasc Biol 15, 1070-1078.

47. Mensink RP, Ebbing S, Lindhout M, et al. (2002) Effects of plant stanol esters supplied in low-fat yoghurt on serum lipids and lipoproteins, non-cholesterol sterols and fat soluble antioxidant concentrations. Atherosclerosis 160, 205-213.

48. Hallikainen MA, Sarkkinen ES \& Uusitupa MI (2000) Plant stanol esters affect serum cholesterol concentrations of hypercholesterolemic men and women in a dosedependent manner. J Nutr 130, 767-776.

49. Denke MA (1995) Lack of efficacy of low-dose sitostanol therapy as an adjunct to a cholesterol-lowering diet in men with moderate hypercholesterolemia. Am J Clin Nutr 61, 392-396.

50. Jones PJ, Vanstone CA, Raeini-Sarjaz M, et al. (2003) Phytosterols in low- and nonfat beverages as part of a controlled diet fail to lower plasma lipid levels. J Lipid Res 44, 1713-1719.

51. AbuMweis SS, Vanstone CA, Ebine N, et al. (2006) Intake of a single morning dose of standard and novel plant sterol preparations for 4 weeks does not dramatically affect plasma lipid concentrations in humans. J Nutr 136, 1012-1016.

52. Noakes M, Clifton PM, Doornbos AM, et al. (2005) Plant sterol ester-enriched milk and yoghurt effectively reduce serum cholesterol in modestly hypercholesterolemic subjects. Eur J Nutr 44, 214-222.

53. Nestel P, Cehun M, Pomeroy S, et al. (2001) Cholesterollowering effects of plant sterol esters and non-esterified stanols in margarine, butter and low-fat foods. Eur J Clin Nutr 55, 1084-1090.

54. Niittynen LH, Jauhiainen TA, Poussa TA, et al. (2008) Effects of yoghurt enriched with free plant sterols on the levels of serum lipids and plant sterols in moderately hypercholesterolaemic subjects on a high-fat diet. Int J Food Sci Nutr 59, 357-367.

55. Doornbos AM, Meynen EM, Duchateau GS, et al. (2006) Intake occasion affects the serum cholesterol lowering of a plant sterol-enriched single-dose yoghurt drink in mildly hypercholesterolaemic subjects. Eur J Clin Nutr 60, 325-333. 
56. Plat J, van Onselen EN, van Heugten MM, et al. (2000) Effects on serum lipids, lipoproteins and fat soluble antioxidant concentrations of consumption frequency of margarines and shortenings enriched with plant stanol esters. Eur J Clin Nutr 54, 671-677.

57. Matvienko OA, Lewis DS, Swanson M, et al. (2002) A single daily dose of soybean phytosterols in ground beef decreases serum total cholesterol and LDL cholesterol in young, mildly hypercholesterolemic men. Am J Clin Nutr 76, 57-64.

58. Weststrate JA \& Meijer GW (1998) Plant sterol-enriched margarines and reduction of plasma total- and LDLcholesterol concentrations in normocholesterolaemic and mildly hypercholesterolaemic subjects. Eur J Clin Nutr 52, 334-343.

59. Plat J \& Mensink RP (2000) Vegetable oil based versus wood based stanol ester mixtures: effects on serum lipids and hemostatic factors in non-hypercholesterolemic subjects. Atherosclerosis 148, 101-112.

60. Neil HA, Meijer GW \& Roe LS (2001) Randomised controlled trial of use by hypercholesterolaemic patients of a vegetable oil sterol-enriched fat spread. Atherosclerosis 156, 329-337.

61. Jones PJ \& Schoeller DA (1990) Evidence for diurnal periodicity in human cholesterol synthesis. J Lipid Res 31, 667-673.

62. Jones PJ, Pappu AS, Illingworth DR, et al. (1992) Correspondence between plasma mevalonic acid levels and deuterium uptake in measuring human cholesterol synthesis. Eur J Clin Invest 22, 609-613.

63. Cella LK, Van Cauter E \& Schoeller DA (1995) Effect of meal timing on diurnal rhythm of human cholesterol synthesis. Am J Physiol 269, E878-E883.
64. AbuMweis SS, Vanstone CA, Lichtenstein AH, et al. (2009) Plant sterol consumption frequency affects plasma lipid levels and cholesterol kinetics in humans. Eur J Clin Nutr 63 , 747-755.

65. Hyun YJ, Kim OY, Kang JB, et al. (2005) Plant stanol esters in low-fat yogurt reduces total and low-density lipoprotein cholesterol and low-density lipoprotein oxidation in normocholesterolemic and mildly hypercholesterolemic subjects. Nutr Res 25, 743-753.

66. Weidner C, Krempf M, Bard JM, et al. (2008) Cholesterol lowering effect of a soy drink enriched with plant sterols in a French population with moderate hypercholesterolemia. Lipids Health Dis 7, 35.

67. Mussner MJ, Parhofer KG, Von Bergmann K, et al. (2002) Effects of phytosterol ester-enriched margarine on plasma lipoproteins in mild to moderate hypercholesterolemia are related to basal cholesterol and fat intake. Metabolism $\mathbf{5 1}$, 189-194.

68. Naumann E, Plat J \& Mensink RP (2003) Changes in serum concentrations of noncholesterol sterols and lipoproteins in healthy subjects do not depend on the ratio of plant sterols to stanols in the diet. $J$ Nutr 133, 2741-2747.

69. Seppo L, Jauhiainen T, Nevala R, et al. (2007) Plant stanol esters in low-fat milk products lower serum total and LDL cholesterol. Eur J Nutr 46, 111-117.

70. Hegele RA, Wang J, Harris SB, et al. (2001) Variable association between genetic variation in the CYP7 gene promoter and plasma lipoproteins in three Canadian populations. Atherosclerosis 154, 579-587.

71. Hegele RA, Brunt JH \& Connelly PW (1995) Multiple genetic determinants of variation of plasma lipoproteins in Alberta Hutterites. Arterioscler Thromb Vasc Biol 15, 861-871. 